\title{
Pancreatic Neuroendocrine Tumors: A Literature Review
}

\author{
Amanda Gomes ${ }^{1}$, Rosa Andrea Nogueira Laiso², Luciana Knop ${ }^{3}$, Monique Gonçalves Alves ${ }^{1,2}$ \\ Laertty Garcia de Sousa Cabral1 ${ }^{2}$, Durvanei Augusto Maria ${ }^{1,2^{*}}$ \\ ${ }^{1}$ Medicine School of University of São Paulo; ${ }^{2}$ Laboratory of Development and Innovation, Butantan Insitutite; São Paulo, \\ São Paulo; ${ }^{3}$ ISI-SENAI-CIMATEC; Salvador, Bahia; Brazil
}

\begin{abstract}
Pancreatic neuroendocrine tumors (PNETs) affect 1\%-3\% of patients with pancreatic cancer. This tumor is rare, difficult to diagnose, and clinically laborious. They have an estimated incidence of up to 1 case per 100,000 inhabitants every year. Up on diagnosis, most PNETs are considered malignant, with low healing potential, lesions that are generally unresectable, and a metastasis rate of aproximately $50 \%$. PNETs are classified as functional and non-functional. The tumor functional produces hormones such as gastrin, insulin, somatostatin, glucagon, among others. They are symptomatic due to hormonal hypersecretion and occur in $30 \%$ of cases. The other $70 \%$ are non-functioning, and despite producing a series of substances and some hormones such as beta HCG and alpha HCG, they are silent tumors, with no significant clinical syndrome. The present study presented scientific evidence about PNETs, the types of pancreas endocrine-tissue tumors, the rate of survival, diagnosis, treatments, and prognosis, to provide solid support to professionals, and contribute to effective decision-making in search of the best clinical outcome.

Keywords: Neuroendocrine Tumor. Pancreatic Tumor. Functioning Pancreatic Tumor.

Abbreviations: NET: Neuroendocrine Tumor. PNET: Pancreatic Neuroendocrine Tumor. MEN-1: Multiple Endocrine Neoplasm type 1. VHL: Von Hippel-Lindau disease. NF-1: Neurofibromatosis type 1. F-PNET: Functioning Pancreatic Neuroendocrine Tumor. NF-PNET: Non-Functioning Pancreatic Neuroendocrine Tumor. WHO: World Health Organization. TNM: Classification System for Malignant Tumors. AJCC: American Joint Committee on Cancer. UICC: Union for International Cancer Control .
\end{abstract}

\section{Introduction}

Pancreatic neuroendocrine tumors (PNETs) affect $1 \%-3 \%$ of patients with pancreatic cancer. They are considered a rare tumor, difficult to diagnose, and clinical practice. The incidence is up to 1 case per 100,000 inhabitants every year [14]. Studied necropsies provide data that contribute to the prevalence of PNETs $(0.5 \%-10 \%)[3,4]$. Upon diagnosis, most PNETs are considered malignant, with low healing potential. The lesions are generally unresectable, and the metastasis rate is $\geq 50 \%$ [5]. PNETs are classified as functional and non-functional. Those that are functional

Received on 12 June 2020; revised 19 July 2020.

Address for correspondence: Prof. Dr. Durvanei Augusto Maria. Instituto Butantan, Laboratório de Desenvolvimento e Inovação, Avenida Vital Brasil, Número 1500, CEP: 05503 900, São Paulo, SP, Brasil. Fone/Fax: (5511)2627-9750. E-mail: durvanei@usp.br.

J Bioeng. Tech. Appl. Health

2020;3(3):288-297.

(C) 2020 by SENAI CIMATEC. All rights reserved. produce hormones such as gastrin, insulin, somatostatin, glucagon, among others, and they are symptomatic due to hormonal hypersecretion. Their prevalence is $30 \%$ of the PNETs' cases. The non-functional tumors (70\% of the PNETs' cases) are silent with no significant clinical symptoms, despite the production of substances and some hormones such as beta and alpha HCG [2,3].

Koo and colleagues [6] showed that metastasis of PNETs can be discovered before primary tumors. The proposal of the study was based on the use of PABX8 markers and Islets 1, which was used for immunohistochemical tests of pancreatic origin's NETs. The study included 110 samples of primary PNETs and 73 of NETs, of which 28 were pancreatic, 5 pulmonary, 37 ileum, 1 rectal, 1 colon, and 1 duodenal. The results showed that Islets 1 had $68 \%$ of specificity for metastatic PNET, revealing that it can be used for investigation of this tumor; and $\mathrm{PABX} 8$ remains more specific as a primary PNET marker, with $88 \%$ of primary PNET samples detected by this marker [6]. 
PNETs are uncommon and without a germline-associated mutation. KRAS gene mutations, commonly found (somatic) in pancreatic adenocarcinoma, are almost completely absent in pancreatic NETs. Genetic syndromes such as multiple endocrine neoplasia type 1 (MEN-1), Von HippelLindau disease (VHL), neurofibromatosis type 1 (NF-1), Mahvash's disease, and tuberous sclerosis (TS) have been correlated with the development of NETs in several studies. Around $12 \%$ of patients with VHL develop PNET, while NF-1 and TS have been reported casually. However, there isn't much data due to their rareness. On the other hand, most patients with MEN-1 have PNET, and typically numerous microadenomas, which may be associated with the premature death of these patients [7].

So, this study presents scientific evidence about PNETs (the types of pancreas endocrine-tissue tumors, the rate of survival, diagnosis, treatments, and prognosis) to provide solid support to professionals and contribute to effective decisionmaking in search of the best clinical outcome.

\section{Methods}

We carried out a literature review in the academic databases such as Pubmed and Scielo, using the terms "Neuroendocrine Tumor", "Pancreatic Tumor" and "Functioning Pancreatic Tumor", and new technology platforms that select the most cited articles crossing them with high impact journals $(>7.0)$. However, for some references, we used only the most cited articles. We used reviews and cases' studies about the theme, diagnosis, treatment, and survival of patients with Pancreatic Neuroendocrine Tumors. We utilized a few articles published 12 years ago, and we also researched the World Health Organization classifications for NET of pancreatic origin.

\section{Functioning Pancreatic Neuroendocrine Tumors (F-PNETs)}

The functioning PNETs are producers of hormones such as gastrin, insulin, somatostatin, glucagon, among others, and are symptomatic due to hormonal hypersecretion. In order of frequency, PNETs are Insulinomas (Beta cells), Gastrinomas and Glucagonomas (Alpha cells), Somatostinomas (Delta cells), Vipomas (Delta 2 cells) e PPomas (PP cells). Since 2010, according to the World Health Organization (WHO), PNETs should be classified based on cell proliferation and tumor morphology. One of the markers of cell proliferation rate is $\mathrm{Ki}-67$ [2,3,8]. WHO also classifies PNETs as 1) well-differentiated, less aggressive; and 2) poorly differentiated, highly aggressive [2]. When compared to pancreatic adenocarcinomas, whose survival is 5-year $(\sim 6 \%$, varying from $2 \%-9 \%$ ), PNETs are slow-growing and have a better prognosis [3]. In a retrospective study by Sanchez-Bueno and colleagues [4], in which medical records of 95 patients after resection of TNEPs were reviewed, the 5-year survival rate was $100 \%$ for well-differentiated tumors. They considered variables such as age, sex, classification of tumors as sporadic or familial, functioning or non-functioning tumor, type of tumor, location, surgical technique, tumor size, multifocal tumors, and recurrence rate [4]. Concerning the diagnosis of PNETs, positronemitting tomography integrated with computed tomography (PET/CT) associated with radiopharmaceuticals is a promising technique in recent years [9].

\section{Non-Functioning Pancreatic Neuroendocrine Tumors (NF-PNETs)}

NF-PNETs are originated in pancreatic tissues and do not produce any substance capable of causing clinical syndromes. These tumors secrete chromogranin A and B (90\%-100\%), AlphaHCG (Human Chorionic Gonadotropin) (40\%), Neuron Specific Enolase (31\%), as well as BetaHCG (20\%). As 40\%-90\% of NF-PNETs secrete pancreatic peptides called PP, they are also called PPomas [8].

NF-PNETs can additionally secrete ghrelin, neurotensin, calcitonin, and other neurotransmitters. 
This is a silent tumor in which $30 \%-40 \%$ continue to behave with nonspecific symptoms, only being detected after screening for many symptoms, typical of other diseases and syndromes. The manifestations of NF-PNETs are produced internally by the tumor, due to the tumor mass. So patients end up looking for help late, and 64\%-92\% of them already have liver metastasis, and $72 \%$ with tumors $>5 \mathrm{~cm}$. These tumors are commonly solitary, with a small exception of patients with MEN 1, in which the manifestations occur in multiple tumors, mainly in the head of the pancreas. Most NF-PNETs, when compared to F-PNETs, can be considered asymptomatic, since they do not present significant symptoms to characterize the clinical condition of the disease and cannot be differentiated from the functioning pancreatic endocrine tumors by immunohistochemical. Studies show that $80 \%$ $100 \%$ of patients with MEN-1 have microscopic NF-PNETs, and these tumors become large and symptomatic in the minority of cases $(0-13 \%)$. In VHL disease, $12 \%-17 \%$ of patients develop NF-PNETs, and $4 \%$ of them reach $\geq 3 \mathrm{~cm}$. The symptoms commonly presented by patients are abdominal pain (30\%-80\%), jaundice (20\%-35\%), weight loss, fatigue, and bleeding (low proportion) [8].

\section{Classification of Neuroendocrine Pancreatic Tumors}

The prognosis of PNETs can be established according to size, histological findings, and staging. Since 2010, the classification by World Health Organization (WHO) divides PNETs into three degrees (G1, G2, and G3) based on expression Ki-67 of the nuclear antigen $(<2 \%, 2-20 \%$, and $>20 \%)$ and on the rate of mitotic division $(<2 \%$, $2 \%-20 \%$ and $>20 \%$ ): $\mathrm{G} 1$ and $\mathrm{G} 2$ are referred as NETs and G3 as neuroendocrine carcinomas. NETs well and moderately-differentiated (G1/G2) show a higher survival rate when compared to poorly-differentiated neuroendocrine carcinomas (G3). According to the Classification of Malignant Tumors (American Joint Committee on Cancer and Union for International Cancer Control AJCC/UICC), the tumor is classified into T1a $(<1 \mathrm{~cm}), \mathrm{T} 1 \mathrm{~b}(1-2 \mathrm{~cm})$ and T2 $(>2 \mathrm{~cm}) ; \mathrm{T} 3$ and T4 are locally advanced tumors (Table 1) [10].

Histologically, NETs are classified into typical and atypical (anaplastic). Typical tumors are welldifferentiated, composed of a small cell mass, with regular cells and rounded nucleus. Atypical tumors have nuclear atypia and accelerated mitotic activity concerning typical tumors, as well as areas of necrosis. There are five distinct histological patterns: insular, glandular, undifferentiated, differentiated, and mixed (Table 2). Nevertheless, we emphasize that there are exceptions in cellular behavior based on histological characteristics, but it should be considered that some histological characteristics determineNET tumor behavior, such as vascular, lymphatic, and organ wall invasion, degree of cellular atypia, nucleocytoplasmic ratio, presence and extension of tumor necrosis and mitotic index. Markers of cell proliferation (Ki67 Index) and p53 expression are important in determining tumor aggressiveness [11].

\section{Insulinomas}

Insulinomas are tumors characterized by hyperfunction of beta cells that produce insulin in the pancreatic islets. These tumors are intrapancreatic, and extraglandular involvement is rare. Considered the most common PNETs, they correspond to $35 \%-40 \%$ of the endocrine pancreas' lesions $[7,9,12]$. It is a benign tumor in most cases (85\%-99\%), single $(93 \%-98 \%),<25 \mathrm{~mm}$ in diameter. However, $5 \%-12 \%$ of insulinomas are malignant and $>30 \mathrm{~mm}$, and up to one-third of malignant insulinomas have metastases at the time of diagnosis. In cases with multiple tumors (10\%) half of them are associated with MEN-1 $[9,13]$. Insulomonas have a high frequency in females (59\%), they can appear at any age, but they are predominant from 45 to 50 years, except for patients with MEN-1, in which they are commonly diagnosed at 25 years of age. The possibility of diagnosing MEN-1 should be investigated in all 
Table 1. Neuroendocrine tumors classification.

\begin{tabular}{lc}
\hline NET Classification & Ki-67 index \\
\hline Well-differentiated neuroendocrine tumor (also called typical carcinoid) & $<2 \%$ \\
Well-differentiated neuroendocrine carcinoma & $2 \%-15 \%$ \\
Poorly-differentiated neuroendocrine carcinoma & \\
\hline
\end{tabular}

Source: Adapted from Araújo and colleagues [11].

Table 2. Classification AJCC/UICC.

\begin{tabular}{ll}
\hline NET & AJCC/UICC \\
\hline T1 & Confined to the pancreas, $<2 \mathrm{~cm}$ \\
T2 & Confined to the pancreas, $>2 \mathrm{~cm}$ \\
T3 & Peripancreatic spread, but without large vascular invasion (upper celiac or mesenteric vessels) \\
T4 & Major vascular invasion (celiac axis or superior mesenteric artery) \\
\hline
\end{tabular}

Source: Adapted from Braga and colleagues [5].

Source: Adapted from reference Araújo and colleagues [11]

patients with insulinoma [9, 12]. This pancreatic tumor is the most common cause of hypoglycemia - relating to endogenous hyperinsulinism - and the incidence of insulinomas is 1-4 cases per 1 million people [14]. Insulinomas are clinically manifested by the Whipple triad: (1) episodic hypoglycemia with low glycemic values $(40-50 \mathrm{mg} / \mathrm{dL})$ and (2) central nervous system (CNS) dysfunction associated with hypoglycemia (this manifestation may progress to mental confusion, anxiety, stupor, and coma), and (3) such dysfunction can be reversed with the administration of glucose. Caldas and colleagues [9] showed a case study that in addition to the hypoglycemia associated with the Whipple triad, $29 \%$ of the patients exhibited postprandial hypoglycemia, $21 \%$ fasting, and postprandial hypoglycemia, and only $6 \%$ manifested just hypoglycemia. This same study recommends that the diagnosis of insulinoma should consider the patient's clinical history, and not be excluded based only on the moments when hypoglycemia occurred, since the average between the onset of symptoms and the diagnosis is 19-20.9 months [12]. Common autonomic symptoms of insulinoma are diaphoresis, tremor, and palpitations, while neuroglycopenic symptoms include, confusion, behavioral changes, personality changes, visual disturbances, seizures, and coma [7, 14].

As insulinoma patients could have neuropsychiatric symptoms, it is common a diagnostic error (approximately $20 \%$ of cases). This reveals why is important the knowledge of the patient's clinical history avoiding late diagnosis of the disease. In some cases, due to an absence of adrenergic symptoms associated with acute hypoglycemia (confusion, seizures, and coma), the patient with this condition, which is still reversible, ends up in other medical areas not related to the real condition, which compromise the treatment and the best clinical outcome $[9,12]$. So, the diagnosis of insulinomas is based on the clinical history of patients, laboratory confirmation, which implies the demonstration of high levels of insulin (40-55UI/mL), spontaneously or caused by prolonged fasting tests. Approximately $97 \%$ of insulinomas are diagnosed before 45 hours of fasting, despite the protocol following 72 hours. Elevated levels of $\mathrm{C}$ peptide are also considered (average of $4.5 \mathrm{ng} / \mathrm{mL}$ and a maximum of $8.4 \mathrm{ng} / \mathrm{mL}$ ), which allows the exclusion of false hypoglycemia. Imaging tests should be performed after biochemical confirmation, due 
to $80 \%$ of insulinomas have a diameter $<2 \mathrm{~cm}$, which is difficult to be located. The search for the preoperative location should be performed exhaustively before surgical resection to reduce the manipulation of the pancreas during surgery since $10 \%-27 \%$ of insulinomas not preoperatively located are also not located intraoperatively [9, $12,15]$. Echoendoscopy is the most successful method in cases of preoperative localization, associated with computed tomography (CT) with multidetector and intravenous contrast, in which sensitivity can reach up to $100 \%$. However, due to failures in locating insulinomas, other methods for diagnosis are used and improved in studies, such as ultrasound with contrast or scintigraphy marked with peptide 1 Glucagon-like, which has been showing promising data [13].

Surgery is the main treatment for insulinomas, but after resection, the major patients need symptom control and long-term cure. For welldifferentiated primary and metastatic functional PNETs, surgery should always be considered, even when the liver or regional lymph nodes are involved. In the case of unresectable metastatic disease, despite the controversies about the morbidities associated with surgery, such as pancreatic fistula, postoperative hyperglycemia, surgical site infection, late diabetes, among others, the resection of the primary lesion is considered palliative but appropriate to symptomatic control and a better outcome. Studies have suggested improvement of overall long-term survival and reduction of disease progression after resection of the primary tumor in the advanced stage [10, 16]. Surgeons describe several types of surgical approaches; and the most cited is insulinoma enucleation and partial distal pancreatectomy. Currently, laparoscopic resection of insulinoma is frequent due to shorter hospital stay and beneficial results to other similar procedures. Robotic enucleation of insulinoma, although little known, presents good results in the short and long term when compared to the laparoscopic approach [16]. Physicians may try pharmacological treatment before surgery in recurrent cases or for malignant insulinomas. For early insulinomas, it is recommended that changes in diet, treatment with medications such as diazoxide or everolimus, and the use of medical bracelets are prudent steps. Therapeutic alternatives, including glucagon pen, somatostatin analogs, and steroids, are also considered [17]. Somatostatin, produced in pancreatic delta cells, acts via paracrine regulation of insulin and glucagon secretion, also regulating cell proliferation, through interaction with five different types of somatostatin receptors coupled to $\mathrm{G}$ protein, called SSTR1-5. These SSTR subtypes have distinct molecular structures,

Table 3. Procedures for locating insulinomas.

\begin{tabular}{lc}
\hline Procedure & Sensitivity \\
\hline Abdominal Computed Tomography & $70 \%-80 \%$ \\
Preoperative Abdominal Ultrasonography (US) & $13 \%-67 \%$ \\
Abdominal Magnetic Resonance & $\mathrm{Up} \mathrm{to} 85 \%$ \\
111In-pentreteotide Scintigraphy & $50 \%-60 \%$ \\
Dosage of Insulin in the Hepatic Vein after Intra-Arterial & $91 \%-100 \%$ \\
$\quad$ Infusion of Calcium Gluconate & \\
Pancreatic Endoscopic Ultrasonography & $60 \%-90 \%$ \\
Transoperative Pancreatic Ultrasonography & $91 \%$ \\
Transoperative Pancreatic Palpation & $75 \%-90 \%$ \\
US + Transoperative Pancreatic Palpation & $\sim 100 \%$ \\
\hline
\end{tabular}

Source: Adapted from Santos [15]. 
tissue distribution, intracellular signaling, and pharmacological characteristics that inhibit different types of hormones. SSTR2 is the dominant receptor on alpha (glucagon) and beta (insulin) cells [10].

Diazoxide is the appropriate option if the patient has an unresectable tumor, metastatic disease, or if it is a patient to which surgical treatment is not recommended. A significant reduction in the release of insulin and an increase in glycogenolysis is expected with the use of diazoxide. A rate of $60 \%$ of the patients achieves the expected results with symptoms-free. The efficacy of octreotide in patients with refractory hypoglycemia is smaller, but it remains an option for patients who do not respond to diazoxide. For patients with refractory symptoms and stable tumor volume, adjustment of the dose of octreotide, a potent inhibitor of somatostatin, GH, glucagon, and insulin, as well as surgical resection, may be considered [9]. Considering that most of these tumors are of benign origin, surgical resection (laparoscopic or laparotomy) is the treatment of choice with a high rate of cure $(75-98 \%)$. However, the prognosis depends on the stage of the disease and/or if the resection is achieved completely [18].

\section{Gastrinomas}

Gastrinomas, also called Zollinher-Ellison Syndrome (ZES), come from G cells, present in the duodenum, pancreas, and stomach. These tumors ectopically secrete gastrin, causing hypergastrinemia and, consequently, an increase in the secretion of gastric acid, which results in peptic ulcers. Some pathologists classify as gastrinomas some tumors that present gastrin in assays by immunocytochemistry (such as bronchogenic carcinomas, colorectal cancer, among others). However, these tumors, although containing gastrin, are not associated with hypergastrinemia and cannot be recognized as gastrinomas or ZES [19].

The incidence of gastrinomas is $0.5-1.5$ cases per 1 million people per year. They are tumors located in the duodenum $(70 \%)$ or pancreas $(20 \%$ $25 \%$ ). Approximately $80 \%$ are sporadic and $20 \%$ $25 \%$ are associated with MEN-1. More than half of gastrinomas are malignant and have a high potential for infiltrating regional lymph nodes and liver metastases $[19,20]$.

Serum gastrin levels ten times above the normal limit $(>1,000 \mathrm{pg} / \mathrm{mL})$, in the presence of gastric secretion with a $\mathrm{pH}$ below 2 , is suggestive for the diagnosis of gastrinoma. When there are moderate changes in baseline gastrin levels, a secretin test should be performed. For example, an increase in gastrin levels above $120 \mathrm{pg} / \mathrm{mL}$ concerning baseline fasting levels suggests investigation for gastrinoma, with sensitivity and specificity of $94 \%$ and $100 \%$, respectively. Gastrin levels should be measured after discontinuing treatment with PPI proton pump inhibitors (omeprazole, pantoprazole) for at least 5 to 7 days, considering that PPI-induced hypochloridia is one of the most frequent causes of hypergastrinemia. However, in patients with ZES, discontinuation of PPI can cause an abrupt and dangerous recovery of acid secretion, therefore, some authors recommend diagnostic evaluation under the protection of PPI [20].

For diagnostic confirmation, numerous methods of tumor localization are performed, including computed tomography (CT), ultrasound, selective angiography, magnetic resonance, a functional location that measures hormonal gradients, endoscopic ultrasound, and scintigraphy of somatostatin receptors (SRS) using 111 In-DTPADPhe-1-octreotide with a $73 \%$ detection rate for gastrinomas. Recent studies demonstrate that SRS is presently the modality of choice for the diagnosis of both localized and metastatic disease [21]. The initial treatment consists of a drug approach with histamine $\mathrm{H} 2$ blockers or PPI proton pump inhibitors, followed by an imaging exam for a surgical approach. It is impossible to be cured without surgical procedures with radical resection in patients with MEN-1-associated gastrinoma, due to multiple and small tumors and the tendency to metastasis. However, in MEN-1 patients the 
surgical indication remains controversial due to short and long-term complications, which is why a Total Pancreatectomy (TP) is rarely performed [19].

Before the use of $\mathrm{H} 2$ antagonists and PPIs, the surgical procedure sought primarily to control symptoms and prevent sequelae. The surgical approach mainly involved total gastrectomy, vagotomy, or, if it is possible, the resection of the tumor mass. Drug therapy in the treatment of gastrinomas provided adequate management of the patient's clinical condition, changing the role of cancer surgery for localization and resection of the primary and metastatic tumor. In the case of sporadic tumors, the surgical approach is indicated as curative, unless there are other contraindications. Surgical exploration is performed through laparotomy for pancreatic gastrinomas and duodenopancreatectomia for duodenal gastrinomas, in addition to the dissection of regional lymph nodes, if there is involvement. Tumors of the pancreatic head should preferably be enclosed and lesions on the pancreatic body or tail require intermediate or distal pancreatectomy. The Whipple procedure (PD) is restricted to exceptional cases $[5,20]$.

Despite high cure rates, biochemical and morphological recurrences are frequent during follow-up. The improvement in survival due to surgery has only been demonstrated recently because these tumors usually progress very slowly and a long follow-up is required. Survival for patients with gastrinoma and liver metastasis is $20 \%-30 \%$ in 5 years [20].

\section{Glucagonomas}

Glucagonomas are PNETs that originated from the alpha cells of pancreatic islets. Considered as a rare tumor, the approximate incidence is 2.4 cases per 100,000,000 people in America, and only 400 cases have been described in the literature. According to the classification of tumors of the digestive system of the World Health Organization (WHO), Glucagonomas are functioning PNETs, and manifest with typical multiple symptoms, such as weight loss of 10 to $15 \mathrm{~kg}$, diarrhea, diabetes mellitus, venous thromboembolism, migratory necrolytic erythema, rash, steatorrhea, anemia, depression, and neuropsychiatric factors. However, early diagnosis, which could provide curative resection, remains difficult due to the uncommonness of this disease [22, 23, 25]. Glucagonomas similarly affect men and women and are usually large tumors $(>4 \mathrm{~cm})$, whose anatomical site is frequent in the distal part of the organ (body and pancreatic tail). They are commonly diagnosed from ages 40 to 50 years, and $50 \%$ of patients present metastases at the time of diagnosis [22, 23, 26].

Changes in plasma glucagon levels may be associated with the appearance of Glucagonomas, so this may be one of the criteria for diagnosis. Even so, caution is necessary, as slight changes in serum dosage above $150 \mathrm{pg} / \mathrm{mL}$ are also present in other clinical conditions, such as difficulties in absorption, infection, and liver cirrhosis. Studies have shown that mutations in the gene responsible for the glucagon receptor are also associated with elevated serum levels of this hormone and that the use of some drugs such as estrogen replenishers in patients with Protein S deficiency has also been correlated with high levels of glucagon [25, 26]. Despite this, fasting levels above $500 \mathrm{pg} / \mathrm{mL}$ can be considered a warning sign (normal range is 50$150 \mathrm{pg} / \mathrm{mL}$ ) since the diagnosis of glucagonoma requires an elevated serum level of glucagon (500-1000 pg/mL). However, considering the possibility of other conditions present high levels of glucagon, imaging methods are important to confirm the pancreatic tumor [23, 24, 26].

The location of the tumor is performed by computed tomography. Pancreatic islet tumors and metastases are usually hypervascular with better definition during the arterial phase of contrast. Confirmation and staging of the tumor can be performed in all patients by SPECT/ CT OctreoScan with 111In-pentreteotide (scintigraphy with somatostatin receptors - SRS), and more recently with Ga-DotataTE PET/CT. 
Another useful of scintigraphy is to evaluate the functional status of the tumor and the secondary location, providing appropriate therapy with peptide receptor radionuclide (PRRT). Treatment may vary depending on the stage of the disease, but currently, surgical resection is the only one that presents a cure chance for glucagonoma (NME can disappear within 1 week after surgery) $[22,25]$. Somatostatin analogs and amino acid solution infusion result just in symptom relief. Other therapies considered useful are transarterial chemoembolization, radiation therapy, and peptide receptor radioligand therapy. Patients may feel significant improvement after treatment and cognitive improvement after surgery, suggesting that neuropsychiatric symptoms are due to the high level of glucagon $[25,26]$. Despite the malignancy of the disease, overall survival even in patients with metastasis ranges from 3 to 8 years [22]. A patient with glucagonoma may have Necrotic Migratory Erythema (NME), characteristic of excess glucagon serum. Glucagonoma syndrome, when not considered therapeutically as a pseudoglucagonoma syndrome, maybe a tumor in the alpha cells of the islets of Langerhans, and the investigation for glucagonoma is recommended when the patient has NME $[23,24,26]$. The mechanisms of glucagonoma-related changes and disorders have yet to be elucidated, but studies show that $76-94 \%$ of patients with glucagonoma can develop diabetes mellitus and $20 \%$ of patients have neurological and/or psychiatric disorders such as dementia, psychosis, agitation, paranoid delusions, ataxia, and hyperreflexia $[23,24,26]$.

\section{Somatostatinomas}

Somatostatinomas are rare tumors with an incidence of 1 case per 40 million people. These tumors originate from the $\mathrm{D}$ cells of the apud system (endocrine cells) can develop in the tissues of the pancreas and duodenum. About 56\%-70\% of somatostatinomas are from pancreatic tissues, $36 \%$ in the head of the organ, $14 \%$ in the body, and $32 \%$ in the pancreatic tail [27]. Somatostatinomas can be functional or non-functional NETs. Duodenal somatostatinomas are commonly non-functional and pancreatic ones are often secretory, also known as functional tumors, in which clinical effects are linked to somatostatin secretion. Somatostatinomas are often malignant (90\%), and approximately $30 \%$ of the duodenal ones and $70 \%-88 \%$ of the pancreatic ones present metastases at the time of diagnosis. Around $40 \%$ of the metastases are hepatic and $30 \%$ in the lymph nodes. Pancreatic somatostatinomas are most often larger than duodenal ones, with an average size ranging between 0.3 and $6 \mathrm{~cm}$ $[27,30]$. The duodenal somatostatinomas are commonly associated with nonspecific symptoms, with neurofibromatosis and less frequently with somatostatinoma syndrome or metastasis [27]. Symptoms are not typical and can be confounded with other diseases such as diabetes, indigestion, and cholelithiasis since when the tumor is secretory, patients have somatostatinoma syndrome, characterized by diabetes, gallstones, and steatorrhea, which are similar to other diseases. The diagnosis is confirmed by histological and immunohistochemical studies and by the presence of specific hormones [30]. If the tumor has a duodenal origin, the diagnosis of somatostatinomas can be performed through upper digestive endoscopy followed by a biopsy, anatomopathological, and immunohistochemical examination. For pancreatic somatostatinomas, the most used methods of diagnosis are computed tomography, ultrasound (US), and nuclear magnetic resonance associated with increased serum somatostatin levels $(>2.5 \mathrm{pg} / \mathrm{mL}$ in $80 \%$ of cases), and serum levels of Chromogranin A and Pancreatic Polypeptide used as nonspecific markers of PNETs $(50 \%-80 \%$ of cases). The endoscopic US can be used to know the extension of the lesion and the locoregional lymph nodes involvement. The somatostatinomas are well located and present in solitary nodules with an average diameter of $5 \mathrm{~cm}$. In general, diagnosis is confirmed through anatomopathological and immunohistochemical examination, with the use 
of chromogranin A, neuron-specific enolase, and synaptophysin as immunohistochemical markers. Approximately $65 \%$ of cases allow for complete resection $[29,30]$. Since most somatostatinomas are located in the periampullary duodenum or the head of the pancreas, a partial pancreatoduodenectomy that preserves the pylorus is the resection performed in patients with somatostatinoma, although total pancreatoduodenectomy may be necessary. As a high proportion of patients have metastases, the role of clearance must be carefully analyzed [30]. Despite surgical represents the only option with curative chances, palliative patients have benefits from surgery for pain relief, clearance of the biliary and intestinal tract, and control of symptoms associated with high serum levels of somatostatin in functional tumors. Chemotherapy is a treatment option for symptom control, trying therapeutic benefits in cases where curative or palliative surgery is not possible [29].

Somatostatin analogs are used to relieve hormonal symptoms, but in severe disease, therapeutic options are limited. Results obtained by Noda and colleagues [4] reveal that the gamma subunit of the high conductance calcium channels (BKCa2) (expressed in many tumorcells such as ovarian tumors, osteosarcomas, breast tumors, and gliomas), whose function is to modulate proliferation, migration, and metastasis, are strongly involved in the proliferation of human somatostatinoma cells, which can suggest a new therapeutic target $[4,28]$. The five-year survival rate for patients with pancreatic and periampullary somatostatinomas is $60 \%-100 \%$ in cases of localized disease, and $15 \%-60 \%$ with metastatic disease. When total resection is achieved, in both cases the five-year survival can reach $100 \%$. Extensive $(>3 \mathrm{~cm})$, poorly differentiated tumors, and lymph node involvement are poor prognostic markers. In $75 \%$ of cases, tumors $>3 \mathrm{~cm}$ have hidden hepatic metastasis, contributing to the poor prognosis. Non-functional and poorly differentiated tumors have a worse prognosis than functional somatostatinomas $[29,30]$.

\section{Final Considerations}

In this article, we demonstrate the difficulty in the management of patients with pancreatic NETs, due to the late diagnosis, which contributes to low patient survival and impaired quality of life. However, it is possible to achieve significant gains based on the data and improvement of progressionfree survival in some cases. The slow development of these tumors and an early diagnosis is possible through careful investigation of patients' clinical history, family history, the use of biochemical exams such as serum levels associated with symptoms, and advanced imaging methods, as well as immunohistochemical analysis and exploratory surgery. Also, the presence of other diseases and comorbidities related to pancreatic NETs should be done.

\section{References}

1. Rawla P, Sunkara T, Gaduputi V. Epidemiology of Pancreatic Cancer: Global Trends, Etiology and Risk Factors. World J Oncol. 2019;10(1):10-27.

2. Belotto, M.; Crouzillard BNS, Araújo KO et al. Tumores Neuroendócrinos Ressecáveis de Pâncreas: Abordagem Cirúrgica. ABCD Arq Bras Cir Dig. 2019, 32(1):e1428.

3. Sanchez-Buenoa F, Gonzalez JMR, Salmero 'GT, et al. Fatores Prognósticos dos Tumores e Pâncreas Ressecáveis. Experiência em 95 pacientes. Cir Esp. 2016, 94(8):473-480.

4. Noda S, Chicazawa K, Suzuki Y et al. Involvement of the g1 Subunit of the Large-Conductance Ca2pActivated $\mathrm{Kp}$ Channel in the Proliferation of Human Somatostatinoma Cells. Biochemical and Biophysical Research Communications. 2020, 525(4):1032-1037.

5. Braga TL, Santos-Oliveira R. PPoma Review: Epidemiology, Aetiopathogenesis, Prognosis and Treatment. Multidisciplinary Digital Publishing Institute: Diseases. 2018, 6(1): 8.

6. Koo J, Mertens RBM, Mirocha JMM et al. Value of Islet 1 and PAX8 in Identifying Metastatic Neuroendocrine Tumors of Pancreatic Origin. Modern Pathology. 2012, 25(2):893-901.

7. Parbhu SK, Adler DG. Pancreatic Neuroendocrine Tumors: Contemporary Diagnosis and Management, Hospital Practice. 2016, 44(3): 109-119.

8. Jameson JL, Fauci AS, Kasper DL et al. Medicina Interna de Harrison. Vol. 2, Edição 20. Porto Alegre, 2020. Cap. 79. 
9. Caldas AR, Teixeira S, Giestas A et al. Insulinoma Pancreático: Casuística de um Hospital Central e Revisão da Literatura. Rev Port Endocrinol Diabetes Metab. 2016, 11(2):181-187.

10. Brown E, Watkin D, Jonathan E et al. Multidisciplinary Management of Refractory Insulinomas. Clinical Endocrinology. 2018, 88 (5): 615 - 624.

11. Araújo NAA, Pantaroto A, Oliveira CT. Tumores Neuroendócrinos: Revisão de Literatura. Perspectivas Médicas. 2012, 23(1): 35-41.

12. Bairrão $\mathrm{M}$, Saraiva $\mathrm{S}$, Viveiros $\mathrm{V}$. Insulinoma e Manifestações Neuropsiquiátricas: A Propósito de Um Caso Clínico. PsiLogos, 2015, 13(2): 32-39.

13. Marques IN, Graça A, Lopes AD et al. Ecoendoscopia no Diagnóstico do Insulinoma. J Port Gastrenterol. 2011, 18(4): 193-195.

14. Okabayashi T, Shima Y, Sumiyoshi T et al. Diagnosis and Management of Insulinoma. World J Gastroenterol. 2013, 19(6): 829-837.

15. Santos MFM. Insulina como Diagnóstico Diferencial da Síndrome Hipoglicêmica: Relato de Caso e Revisão da Literatura. 34f. Trabalho de Conclusão de Residência em Clínica Médica. Hospital do Servidor Público Municipal II, São Paulo, 2015.

16. Bonato FT, Coelho JCU, Petruzzielo A et al. Tratamento Cirúrgico dos Insulinomas do Pâncreas. ABCD Arq Bras Cir Dig. 2012, 25(2):101-104.

17. Nishida JK, Nassar V, Vieira MLH. Processo Interativo para Aferição de Sinais Vitais de Pacientes: Proposta de uma Pulseira Multiparamétrica. Ergodesing \& HCI. 2016, Número especial (4): 83-89.

18. Carvalho $\mathrm{R}$, Branquinho $\mathrm{F}$, Alves $\mathrm{N}$ et al. Insulinoma: A Propósito de um Caso Clínico com Revisão da Literatura. Case Reports Medicina Interna. 2010, 17 (21): 99-103.

19. Gong S, Zhi Li Z, Liu XB et al. Gastrinoma in Multiple Endocrine Neoplasia Type 1 After Total Pancreatectomy: A Case Report. Medicine (Baltimore). 2019, 98(50): e18275.
20. Guarnotta V, Martini C, Davi MV et al. The ZollingerEllison Syndrome: is there a Role for Somatostatin Analogues in the Treatment of the Gastrinoma. Endocrine. 2018, 60(1):15-27.

21. Nowosinska E, Buscombe JR. Radiolabelled Somatostatin Analogues for Single-photon Emission Scintigraphy. Hong Kong Col Radiol. 2010, 13:175-80.

22. Corrias G, Horvat N, Monti S et al. Malignant Transformation of Glucagonoma with SPECT/CT In111 OctreoScan Features: A Case Report. Medicine (Baltimore). 2017, 96(50):e9252.

23. Lobo I, Carvalho A, Amaral C et al. Glucagonoma syndrome and necrolytic migratory erythema. Int $\mathrm{J}$ Dermatol. 2010, 49(1):24-9.

24. John AM, Schwartz RA. Glucagonoma Syndrome: A Review and Update on Treatment. Eur Acad Dermatol Venereol. 2016, 30(12):2016-2022.

25. Song $\mathrm{X}$, Zheng $\mathrm{S}$, Yang G. Glucagonoma and the glucagonoma syndrome (Review). Oncology Letters. 2018, 15(3): 2749-2755.

26. Halvorson SAC, Gilbert E, Hopkins SR et al. Putting the Pieces Together: Necrolytic Migratory Erythema and the Glucagonoma Syndrome. J Gen Intern Med. 2013, 28(11): 1525-1529.

27. Masulović D, Stevic R, Filipović A, Micev M et al. Somatostatin-producing duodenal carcinoma: clinicopathological description of a case. hir IugosL. 2013,60(3):61-4.

28. Halfdanarson TR, Rabe KG, Rubin J et al. Pancreatic Neuroendocrine Tumors (PNETs): Incidence, Prognosis and Recent Trend Toward Improved Survival. 2008, Annals of Oncology19(10): 1727-1733.

29. Henriques AC, Mader AMA, Ramos GM et al. Somatostatinoma de duodeno: relato de caso e revisão da literatura. Arquivos Brasileiros de Ciências da Saúde, v.33, n. 1, p. 36-9

30. Williamson JML, Thorn CC, D Spalding D et al. Pancreatic and Peripancreatic Somatostatinomas. Ann R Coll Surg Engl. 2011; 93(5): 356-360. 